\title{
Triangulationssensoren für Roboteranwendungen
}

G. Seitz, G. Jahn, H. J. Tiziani

Institut für Technische Optik

Pfaffenwaldring 9

7000 Stuttgart 80

Viele MeB- und Prūfprobleme in Fertigung und Montage sind durch reine Grauwert-Bildverarbeitung nicht befriedigend zu lösen, weil hier nur ein informationsreduziertes zweidimensionales Abbild der real dreidimensionalen Szene als Informationsbasis zur Verfügung steht. Dreidimensionale objektinformation bietet hingegen ein wesentlich höheres Informationspotential und ermōglicht neben vōllig neuen Lōsungsansätzen auch weniger aufwendige Lōsungen von Standardproblemen /1/.

Zur dreidimensionalen Objekterfassung werden punkt- und flăchenhaft arbeitende Verfahren eingesetzt. Punktverfahren gewinnen Abstandsbilder durch zeitsequentielle $\mathrm{MeB}$ punkterfassung, wohingegen flächenhafte Verfahren auf zeitlich paralleler Erfassung von 3-D-Information beruhen $/ 2 /$. Die folgenden Ausführungen beschränken sich ausschlieBlich auf punktorientierte Verfahren. Bild 1 zeigt einige punktorientierte, für Roboteranwendungen geeignete AbstandsmeBverfahren.

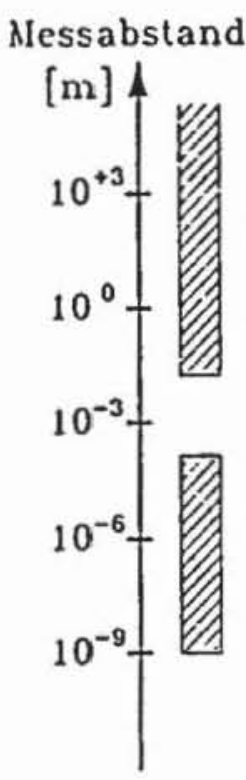

Bild 1: AbstandsmeBverfahren

für Roboteranwendungen

Photometrische Balance-Verfahren basieren auf optischer Pupillen-Triangulation; die Auflösung hăngt also direkt von der Apertur des Meßsystems ab. Da groBe Aperturen im allgemeinen nur bei geringen Objektweiten unter vertretbarem Aufwand realisierbar sind, ist der Anwendungsbereich auf kleine Objektabstände begrenzt.

Laufzeit bzw. PhasenmeBverfahren basieren auf der zeitlichen Vermessung eines modulierten Lichtsignals /3/. Die obere Meßbereichsgrenze wird durch das Signal/Rauschverhältnis der Detektionsmimik bestimmt und kann bei gūnstigen Intensitätsverhält nissen, etwa bei Verwendung von Retroreflektoren, mehrere tausend Kilometer betra. 
gen. Die untere Grenze scheint durch die technischen Möglichkeiten zur Auflösung von Signallaufzeiten im Pikosekundenbereich gegeben zu sein. (Laufzeit für lmm: 6 ps). Der für Roboteranwendungen interessante Bereich zwischen $1 \mu \mathrm{m}$ und $1 \mathrm{~m}$ wird voll durch die optische Triangulation abgedeckt $/ 4 /$.

Eine in diesem Zusammenhang interessante Eigenschaft der Triangulation liegt darin, daB der KompromiB zwischen Auflösung und MeBbereich direkt durch Wahl der Triangulationsbasis vorgegeben werden kann (Bild 2). Die Z-Auflösung der Triangulation ist also unter Annahme eines der Basis entsprechenden MeBabstandes gleich dem doppelten Produkt aus Basis und Winkelauflösung des Detektionssystems. Die in Beispiel angegebene Winkelauflösung wird bei einem CCD-Detektor mit $10 \mu \mathrm{m}$ Pixelperiode und einem AbbildungsmaBstab von ca. 3:1 erreicht, wenn die Punktbildposi-

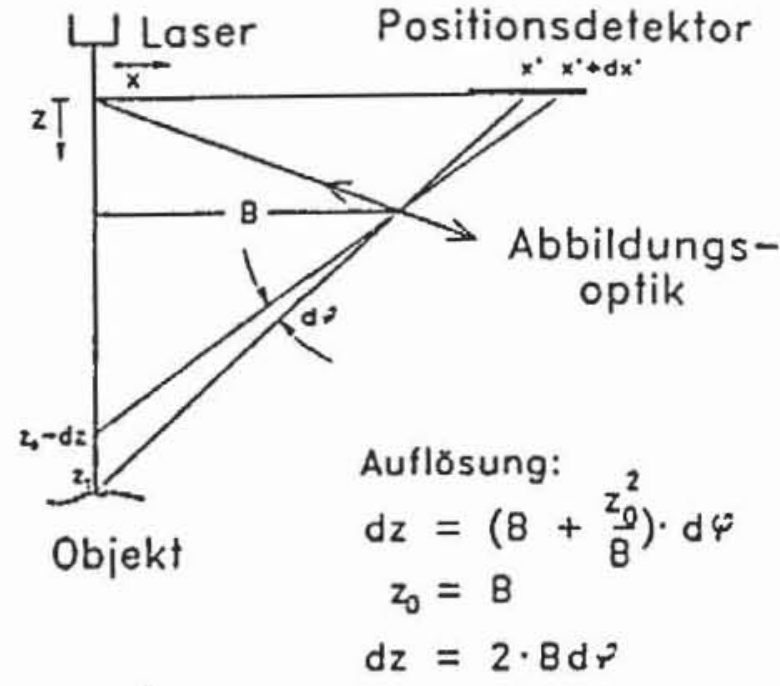

z. B.:

$8=200 \mathrm{~mm}, \quad d r=2 \cdot 10^{-4}, d z=10 \mu \mathrm{m}$

Bild 2: Prinzipskizze

optische Triangulation

tion mit einer Genauigkeit von nur einem Pixel aufgelōst wird.

Das in Bild 2 dargestellte Triangulationsverfahren miBt nur den z-Abstand des Antastpunkts und arbeitet daher eindimensional. Eine mehrdimensionale Erweiterung dieses Verfahrens erfordert, $\mathrm{da} B$ mindestens einer der beiden Strahlengãnge umgelenkt wird. Zur dynamischen Umlenkung optischer Strahlengānge können folgende Prinzipien verwendet werden:

- Spiegelpoiygone

- Akusto-optische Modulatoren

- Galvanometerspiegel

Spiegelpolygone sind mechanisch schnell rotierende Kōrper und können infolge von Kreiselmomenten nur unter groBen Problemen am Roboterarm betrieben werden. Akustooptische Modulatoren sind zwar sehr schnell, arbeiten aber nur in Ablenkbereichen bis ca. $1 \mathrm{Grad}$, was keine ausreichenden MeBfelder zuläßt. Galvanometerscanner erreichen dahingegen Ablenkbereiche von ca. 40 Grad und verursachen keine merklichen Kreiselmomente. Galvanometerscanner scheinen daher momentan die besten Voraussetzun- 
gen zur Verwendung in einem Robot-Sensor zu bieten.

Der einfachste Ansatz zur dreidimensionalen Lasertriangulation ist das in Bild 3 wiedergegebene direkte Triangulationsverfahren: ein über 2 Galvanometerspiegel abgelenkter Laserstrahl markiert einen Oberflächenpunkt $P$, welcher dann auf ein Kameratarget abgebildet wird. Aus den Stellwinkeln der Galvanometerscanner sowie den Koordinaten des Bildpunktes $P^{\prime}$ folgen die 3 räumlichen Koordinaten des Antastpunkts $P$. Zur Positionsdetektion können neben CCD-Kameratargets auch Lateraleffektdioden verwendet werden. Verglichen mit CCD-Kameratargets erreichen Lateraleffektdioden wesent1ich höhere Geschwindigkeiten. Lateraleffektdioden haben jedoch den Nachteil, daB ein Signal erzeugt wird, welches den Intensitātsschwerpunkt des Gesamtbildes wiedergibt. Existieren also neben dem Punkt $P^{\prime}$ noch weitere, etwa durch Umgebungslicht verursachte Bildmerkmale, so erfolgt eine Verfälschung der Punktkoordinate. Schmalbandige, auf die Wellenlänge des Antastlasers abgestimmte Interferenzfilter reduzieren zwar diesen Effekt, bieten jedoch keine Abschirmung gegen sekundäres Streulicht. Eine ausreichende Störsignalunterdrückung scheint nur bei Verwendung ortsauflōsender Sensoren möglich, wo durch eine nachgeschaltete Signalverarbeitung Sekundärreflexionen aufgelöst und anhand einfacher Merkmale wie z. B. geringerer Signalamplitude eliminiert werden können. Zweidimensionale CCD-Arrays sind augenblicklich mit bis zu 2000 * 2000 Pixeln erhältlich und bieten daher eine ausreichende Auflōsung. Die Bildfrequenzen dieser CCD-Sensoren sind jedoch mit ca. 6 Bildern/Sekunde sehr niedrig. Im Unterschied hierzu arbeiten HochgeschwindigkeitsCCD-Targets mit Bildfrequenzen von mehreren $\mathrm{KHz}$ bei Pixelzahlen in der Größenordnung von 250 * 250 Pixeln, was wiederum keine ausreichende Auflōsung gewăhrleistet.

Berücksichtigt man nun, daB die

Triangulation auf der Vermessung eines ebenen Dreiecks beruht, dessen Neigung über den Stellwinkel des Galvanometerscanners 2 (Bild 3) bekannt ist, so wird $k l a r, d a B$ die $y$-Koordinate des Punktbildes $P^{\prime}$ zur Bestimmung der Antastkoordinaten überhaupt nicht erforderlich ist. Das CCD-Kameratarget könnte also durch eine CCD-Zeile ersetzt werden, wodurch eine hohe Meßgeschwindigkeit bei gleichzeitig hoher Auflösung erzielt werden kann. Voraussetzung hierfür ist, $\mathrm{da} B$ das Punktbild auf die Zeile abgebildet wird. Dies kann durch eine anamor-

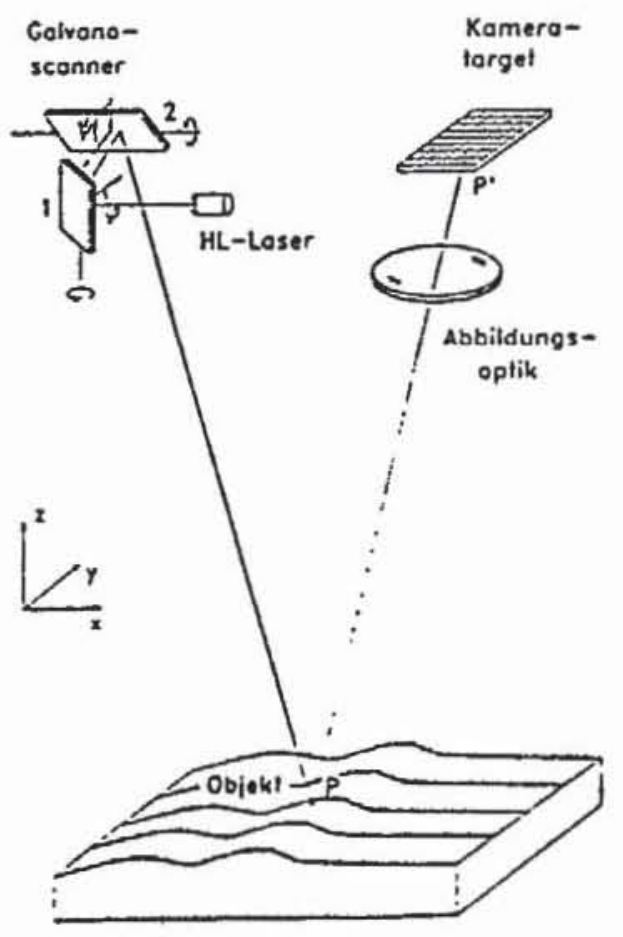

Bild 3: Direkte Lasertriangulation 
photische Optik näherungsweise erreicht werden (Bild 4).

Die in Bild 4 gezeigte Anordnung wurde in einen Roboterkopf (Bild 5) eingebaut und wird zur Vollständigkeitsprüfung elektronischer Leiterplatten eingesetzt. Erreicht werden folgende Leistungsdaten:

- Antastvolumen: $100 * 70 * 100 \mathrm{~mm}^{3}$

- Meßabstand: $200 \mathrm{~mm}$

- Auflösung: $0.1 \mathrm{~mm}$

- Meßzeit: > $\quad>1 \mathrm{~ms} /$ Punkt

Die Meßzeit ist variabel und wird entsprechend den Intensitätsverhältnissen des Antastpunkts eingestellt.

Der Meßbereich des beschriebenen direkten Triangulationsverfahrens wird durch Schärfentiefeprobleme bei der Abbildung eingeschränkt. Da die Beleuchtungsstärke in der Bildebene etwa quadratisch mit dem Abstand des MeBobjekts von der Fokusebene abnimmt, ist der TiefenmeBbereich dieses direkten Triangulationsverfahrens verhältnismäßig klein; das Verfahren eignet sich also vornehmlich für Objekte mit geringer z-Ausdehnung.

Ein erweiterter z-Meßbereich kann durch verbesserte abbildungsseitige Schärfentiefenverhältnisse erreicht werden. Durch geeignete Anordnung von Antaststrah1, Detektor und Abbildungssystem kann die Scheimpflug-Bedingung erfül1t werden (Bild 6). Hierdurch wird eine scharfe Abbildung des Antastpunkts über den gesamten Meßbereich aufkosten einer konstanten Vergrößerung gewährleistet.

Eine Erfüllung der Scheimpflug-Bedingung im zweidimensionalen Fall erfordert nun eine synchrone Ablenkung von Antast-und Abbildungsstrahlengang. Bild 7 zeigt hierzu einen Ansatz: der Laserstrahl wird über einen Galvanometerspiegel abgelenkt, dessen 
Rückseite zur Ablenkung des Abbildungsstrahlengangs verwendet wird. Bei einer Drehung des Galvanometerspiegels folgt das Bildfeld dem Antastpunkt. Laserstrahl und optische Achse der Detektionsoptik schneiden sich bei Durchfahren des Meßbereichs allerdings nicht unter exakt gleichbleibendem Winkel. Da jedoch ein konstanter Winkel zwischen Antaststrahl und optischer Achse notwendige Bedingung zur Erfüllung der Scheimpflug-Bedingung ist, kann diese nicht exakt über den gesamten Meßbereich erfüllt werden.

Einen weiteren Ansatz zeigt Bild 8: Laserstrahl und Abbildungsstrahlengang werden über dieselbe $F 1 a ̈ c h e$ eines Galvanometerspiegels abgelenkt, wodurch der Winkel zwischen Antaststrahi und optischer Achse auch bei Orehen des Galvanometerspiegels konstant bleibt; die Scheimpflug-Bedingung kann also über den gesamten Meßbereich exakt erfüllt werden. Laser-Streulicht, verursacht durch Staubpartikel auf dem Galvanometerspiegel, hat keinen entscheidenden Einfluß auf die Punktdetektion, da dieser Spiegel nahe einer Pupillenebene des Ab-

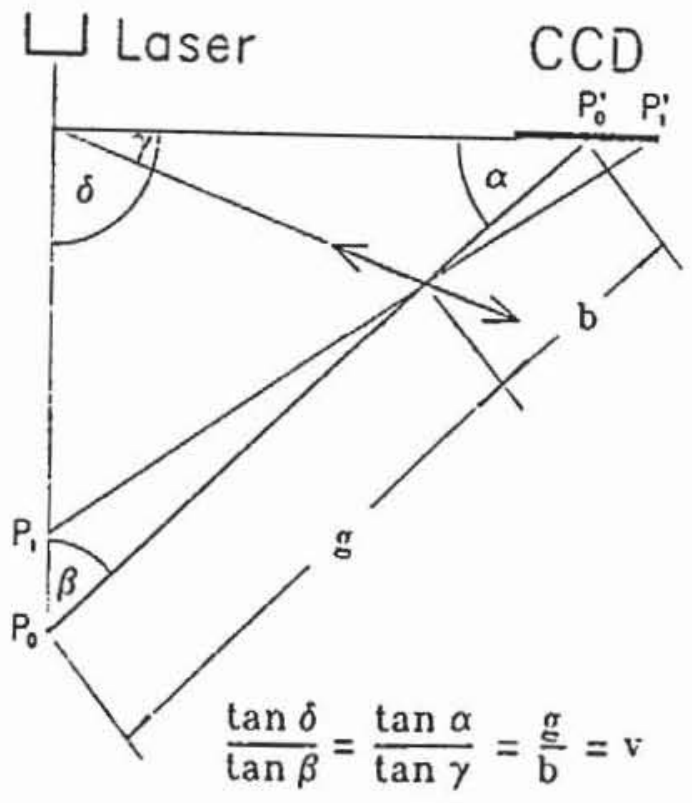

Bild 6: Scheimpflug-Bedingung

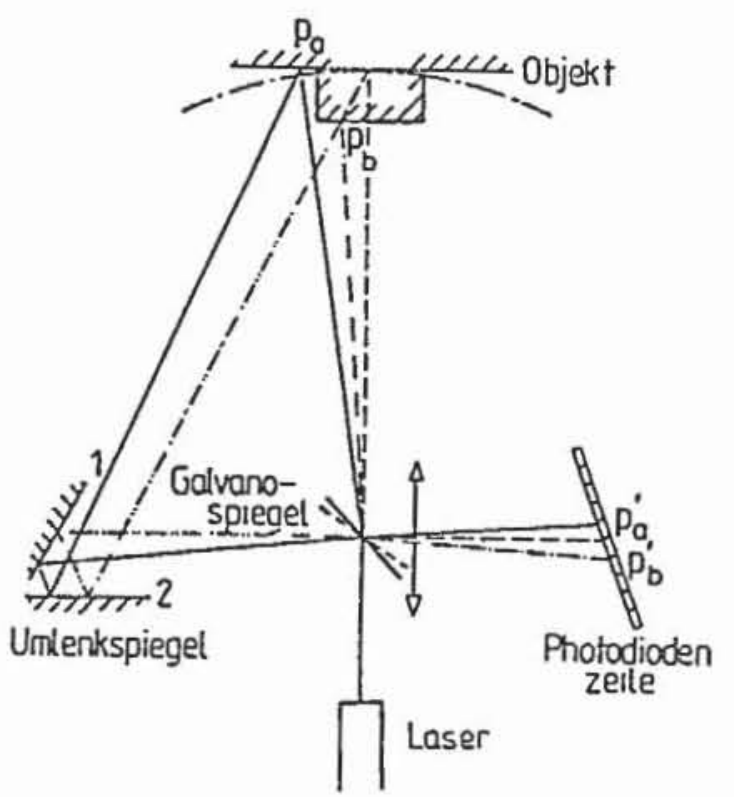

Bild 7: Synchrones Triangulationsverfahren bildungssystems liegt und Pupillenund Bildebene über eine Fouriertransformation zusammenhängen; punktförmige Objekte in der Pupillenebene erhöhen also nur den Gleichlicht-Anteil in der Bildebene.

Diese zweidimensional arbeitende Anordnung kann nun durch Verschieben des Seitenspiegels in Richtung der Galvanometerspiegel-Drehachse um die dritte MeBdimension erweitert werden. Die hierbei auftretende Defokussierung des Punktbilds ist durch Hinzunahme eines entsprechenden Seitenspiegels im Abbildungsstrahlengang zu verhindern (Bild 9). Dieser Spiegel wird mit dem antastseitigen Seitenspiegel starr gekop- 
pelt, wodurch eine exakte Fokussierung des Antastpunktes über den gesamten MeBbereich erreicht werden kann.

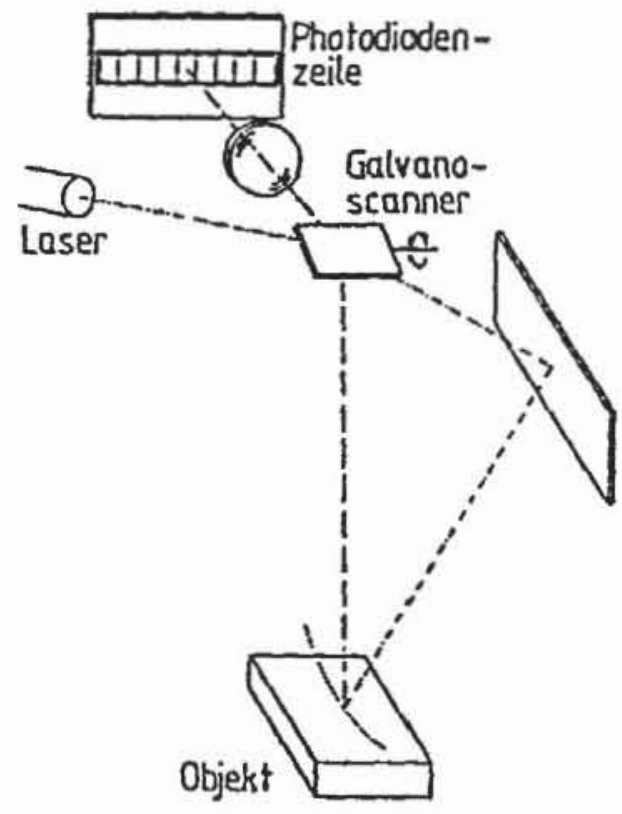

Bild 8: Synchrones Triangulationsverfahren mit verflochtenem Strahlengang

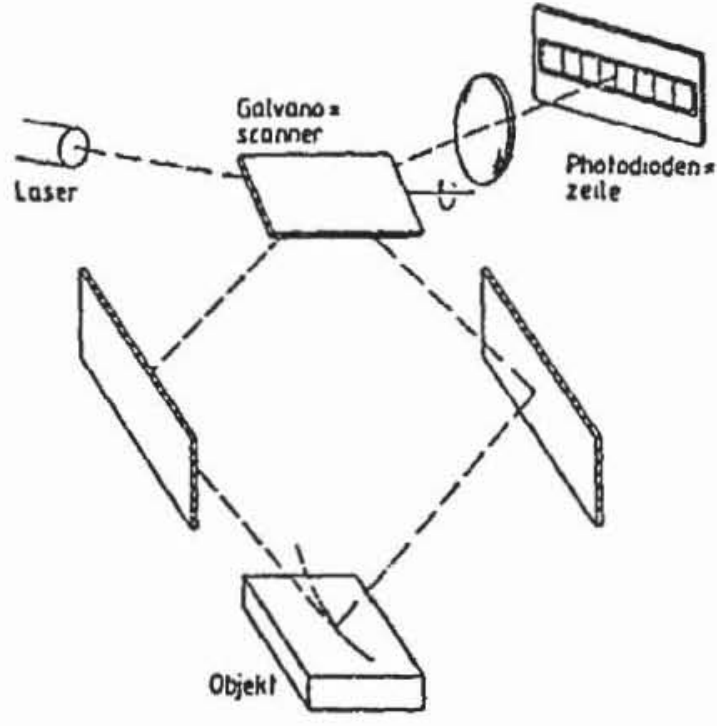

Bild 9: 3-D-Triangulationsverfahren mit verflochtenem Strahiengang

Schrifttum:

/1/ Tiziani, H., J.: Automatisierung der optischen Qualitätsprüfung Technisches Messen Vol. 55, Nr. 12, 1988, S. 481-491

/2/ Jarvis, R., A.: A Perspective on Range Finding Techniques for Computer Vision; IEEE Vol. PAMI-5, Nr. 2, 1983, S. 122-139

13/ Schwarte, R.: Performance Capabilities of Laser Ranging Sensors. Proc. ESA, Workshop on Space Laser Applications and Technology (ESA SP-202, May 1984).

/4/ Seitz, G.; 3-D Koordinatenmessung durch optische Triangulation. Tiziani H. J.; Feinwerktechnik + Messtechnik, Vol. 94, Nr. 7, 1986, Litschel, R.: $\quad$ S. $423-425$ 